\title{
On the Source Field Geometry and Geomagnetic Induction in Southern India
}

\author{
E. Chandra SeKhar and B. R. Arora \\ Indian Institute of Geomagnetism, Colaba, Bombay 400 005, India
}

(Received October 4, 1993; Revised May 31, 1994; Accepted June 2, 1994)

\begin{abstract}
The effect of source field geometry on the response function $(\Delta Z / \Delta H)$, used in geomagnetic depth sounding to decipher lateral conductivity distribution within the earth is highlighted with the help of contour plots of $\Delta Z / \Delta H$ ratios for day and nighttime short-period fluctuations recorded through a largescale magnetometer array operated in the equatorial region of southern India. The representation of the difference between day (non-uniform) and night (uniform) time ratios in terms of the spatial derivatives of source field suggests that the reduction in daytime $\Delta Z / \Delta H$ ratios at stations close to the periphery of the electrojet is due to the mutual balance of external and internal parts in $\Delta Z$. The reduction in daytime $\Delta Z / \Delta H$ ratios near the center of electrojet axis is interpreted to indicate the weakening of the intensity of induced currents due to the presence of second and higher order spatial derivatives in the non-uniform source field.
\end{abstract}

\section{Introduction}

In Magnetotelluric (MT) method, the Tikhonov-Cagniard impedance expressing the relation between orthogonal electric $(E)$ and magnetic $(H)$ field components forms the fundamental response function to yield information on the variation of conductivity with depth. On the other hand in Geomagnetic Depth Sounding (GDS), the transfer functions relating the anomalous vertical field component $(Z)$ to the normal horizontal field components $(D$ and $H$ ) or simply the $\Delta Z / \Delta H$ ratios are used to map the lateral conductivity distribution. In the spatial horizontal gradient (SHG) method, the geomagnetic inductive response function $(C)$ defined as the ratio of vertical field to the gradient of the horizontal components $(C=Z /(\mathrm{d} H / \mathrm{d} x+\mathrm{d} D / \mathrm{d} y))$ leads to the estimate of 1 -D conductivity structures. The traditional interpretation of all these electromagnetic response functions relies on the assumption that the external inducing source field is uniform or at least its scale length is large compared to its depth of penetration (skin-depth) (Schmucker, 1970).

In auroral and equatorial electrojet regions, this assumption of source field uniformity for the period range considered in these investigations (a few mins to $1 \mathrm{hr}$ ) does not always hold. Therefore, the estimation and interpretation of response functions become questionable. The validity of the assumption of plane-wave approximation for a variety of natural sources has been examined by Wait (1954). Giving the modified form of Tikhonov-Cagniard impedance relation, he showed that the correction term is related to the second order spatial derivative in source field. Dimitriev and Berdichevsky (1979) finally pointed out that the principal condition determining the applicability of the 1-D Tikhonov-Cagniard model is the linearity of the horizontal field variations and not their slowness. Corroborating this result, Berdichevsky et al. (1981) showed that in contrast to the MT impedance, modulation of geomagnetic inductive response $(C)$ in SHG is controlled by the third derivative. Progress of theoretical and modeling formulation developed to consider the problem of EM induction by non-uniform sources over a horizontally stratified earth model has been lucidly reviewed by Mareschal (1986). The electromagnetic effects of non-uniform source for the earth model incorporating lateral inhomogeneity has been reported by Mareschal (1981) and Hermance (1984), though the functional correction formulae for GDS response function have not been established.

For the purpose of quantifying the source field effect on induction process, the equatorial regions are 
fortuitously better suited. Firstly because, in those regions, both the uniform and non-uniform fields exist respectively in association with the night and daytime short period fluctuations and secondly that even during daytime the source field geometry does not vary much with local time and magnetic activity, and thus, facilitates to define the source field model reasonably well. Taking the advantage of this situation, the presence of the effect of the electrojet source field on the induction process has been observationally discussed by Papamastorakis and Haerendel (1983) by comparing the $\Delta Z / \Delta H$ ratios of day and nighttime events at permanent Indian equatorial stations Trivandrum (TRD) and Annamalainagar (ANN). Rastogi et al. (1986) and more recently Reddy (1992) mapping the spatial extent of effect of source field on the data of large scale magnetometer array over southern India, have emphasized that non-uniformity affects the strength of induced currents only when the higher order spatial derivatives of the source field are nonzero. Representing the electrojet field by a Gaussian distribution of ionospheric currents, Agarwal and Weaver (1990) have numerically investigated the role of non-uniform source field on electromagnetic induction by estimating the external and internal parts of $H$ and $Z$ components for all stations of southern India array. In this paper we first establish the univocal features of day and nighttime $\Delta Z / \Delta H$ ratios for the stations of south India array using much larger data base than hitherto used in previous studies (Papamastorakis and Haerendel, 1983; Rastogi et al., 1986). Then we attempt to quantify the source field

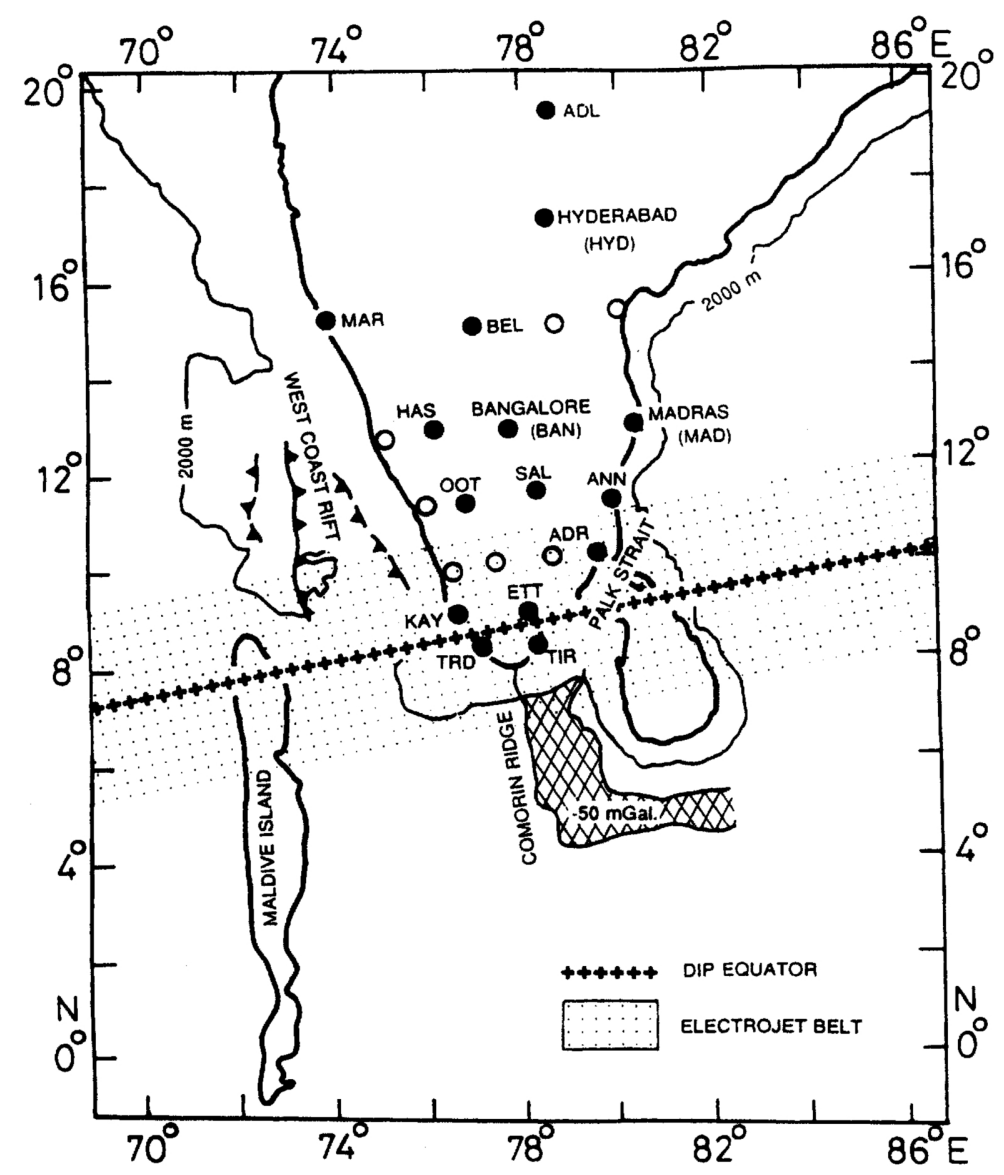

Fig. 1. Location of magnetometer array stations in peninsular India-its major tectonic offshore elements, dip equator and electrojet belt. Solid and open circles represent the location of stations covered under southern India magnetometer array. Data of stations marked by solid circles only were used in the present study. 
effect in terms of spatial derivatives of electrojet field which helps to evaluate their compatibility with theoretically expected behaviour. Owing to different sensitivities of external and internal fields to different orders of spatial derivatives, this representation helps to estimate the control of non-uniformity of the source field on the strength of induced currents and field of external origin.

\section{Basic Data}

For the present study, the magnetic field variations recorded through an array of magnetometers in the equatorial region of peninsular India were utilized. The location of magnetometer array stations in peninsular India with its adjoining tectonic setting, the dip equator and electrojet belt are shown in Fig. 1. The operational details and the steps followed in the reduction of the array data are given in Thakur $e t$ al. $(1981,1986)$. During the ten weeks of the array operation, some five long spells of magnetic storms occurred. But due to the technical problems and large tendency of drift in the suspended sensors of certain Gough-Reitzel magnetometers employed in the survey work, the simultaneity of the data recorded was seriously affected. For the present work, the data of two segments each of 24 -hrs, duration isolated from the magnetic storms of January 28-29, 1980 and February 15-16, 1980 were selected. The starting and ending times of both the selected storm segments are 1500-1459 UT, yielding 1440 values at $1 \mathrm{~min}$ interval in each storm data. The continued sub-storm activity with multiple sequences of short period fluctuations with substantially large amplitudes provided enough events for the statistical formulation adopted here to characterize the source field effects. Secondly, the geographical distribution of the stations, which recorded these events, gave away the requisite spatial coverage right from the dip equator through the periphery of the electrojet to the regions far from the influence of the equatorial electrojet. For the reasons of loss of data, some of the stations used in the earlier investigations of Thakur et al. (1986) could not be included. The later study used the isolated disturbance events and simultaneity of the data though desired, was not essential. In the present case, the character of source field is determined from the spatially distributed data, and the availability of simultaneous data was rather a basic requisite.

The overall behavior of the observed magnetic field variations during storms are the combined effects

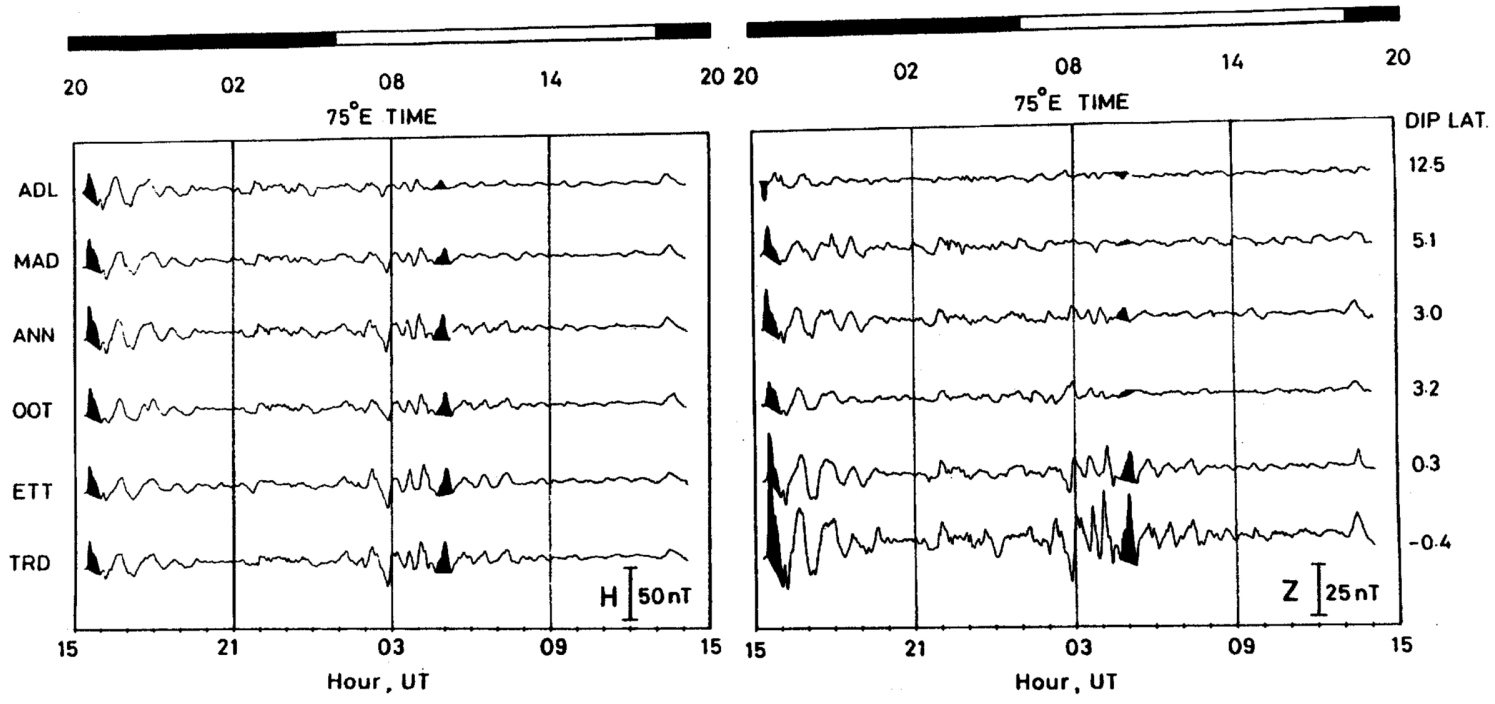

Fig. 2. Short period fluctuations of horizontal $(H)$ and vertical $(Z)$ magnetic field components (after removing long period variations) for a few selected group of stations scanning the latitudinal range covered in the array. The shaded portions in the plots show the example of the significant peaks considered for analysis. 
of regular daily variations, ring current, substorms activity etc. Since the assumption regarding the uniform nature of the source field even for the nighttime variations is valid only for short period fluctuations up to a period range of $60-80 \mathrm{~min}$, the long period variations were approximated by $61-\mathrm{min}$ running average and eliminated from the data. Since in general, the variations in declination $(D)$ are very small in the equatorial region, only the records of horizontal $(H)$ and vertical $(Z)$ magnetic component variations were considered for the present work. Figure 2 gives the stacked plot of short period fluctuations in $H$ and $Z$ components for a group of stations scanning the latitude range covered by the array. Only the short period fluctuations with $H$-peak amplitudes greater than $\pm 7 \mathrm{nT}$ were selected for analysis. This threshold, though somewhat arbitrary, was selected to ensure that the magnitude of accompanying $Z$ fluctuations, where present, were well above the resolution and noise levels of recording. This selection criteria provided some 28 events each from daytime (0600-1800 hrs LT) and nighttime (1801-0559 hrs LT) intervals.

\section{Formulation}

Figures 3(a) and 3(b) give the typical latitudinal distribution of short period fluctuations of $\Delta H$ associated with day and nighttimes respectively. The daytime latitudinal profile of $\Delta H$ shows appreciably enhanced amplitudes at the dip equator. This augmentation is consistent with the concentration of external ionospheric source currents due to high Cowling conductivity. In comparison to this, the nighttime $\Delta H$ values show a fair constancy over the entire latitudinal range. Although the effects of induction in a lateral
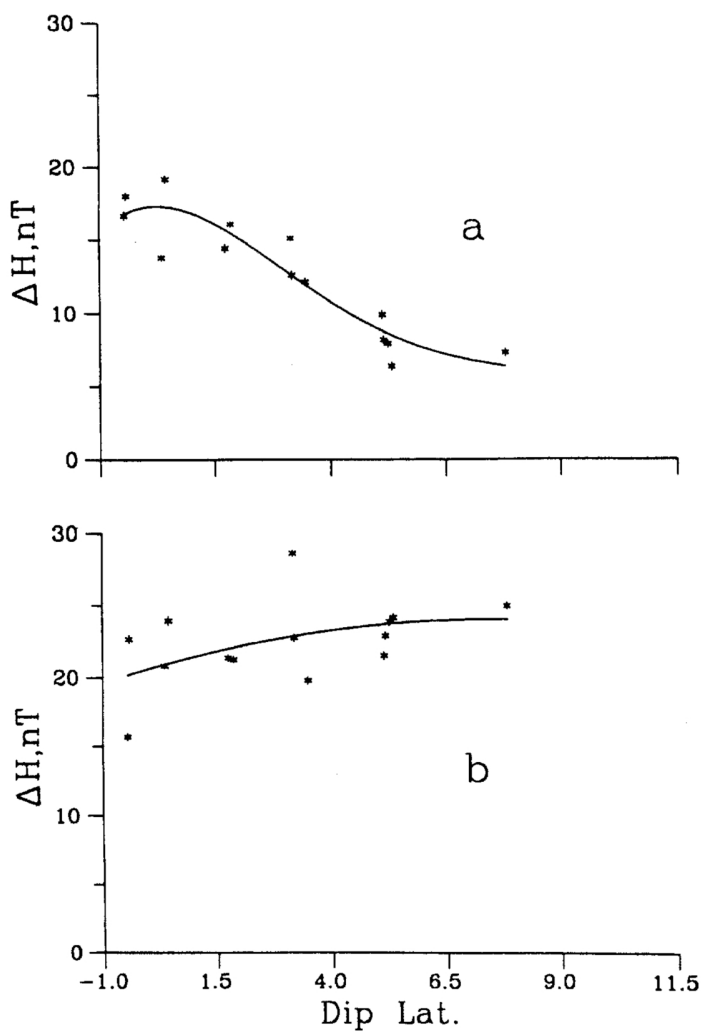

Fig. 3. Latitudinal distribution of short period fluctuations of horizontal magnetic components associated with (a) day and (b) nighttimes for the shaded peaks shown in Fig. 2. 
conductivity inhomogeneity are more conspicuous in $Z$-component, their contributions will always be present in horizontal components. The presence of this anomalous part in $H\left(H_{\mathrm{a}}\right)$ at some stations, being random in relation to the location of dip equator, would be seen as scatter of points around the smooth latitudinal profiles in $\Delta H$. The large scatter of points in Fig. 3 is consistent with the complex distribution of electrical conductivity at the southern tip of India (Ramaswamy et al., 1985; Thakur et al., 1986; Mareschal et al., 1987; Agarwal and Weaver, 1989). The little reduction in $\Delta H$ near the dip equator is more likely to be a manifestation of coast effect (Singh et al., 1982) at TRD, TIR, KAY rather than reflecting a systematic latitudinal progression (Rastogi et al., 1986). Withstanding to the scatter in the plots, the smooth curves in Fig. 3 clearly bring out the non-uniform and uniform character of the source field during day and nighttimes respectively.

The short period fluctuations in $Z$, at night hours show a large variability from station to station, even when the corresponding fluctuations in $H$ have more or less constant amplitude. This variability does not bring out any simple latitudinal behavior. This corroborates the view that fluctuations in $Z$ associated with uniform source are determined by the internal induced currents whose flow pattern is controlled by the lateral conductivity distribution. Under this basic assumption, the observed nighttime perturbations, $\left(\Delta Z_{\mathrm{n}}\right)$ can be considered to be entirely of internal origin $\left(\Delta Z_{i}\right)$, i.e.,

$$
\Delta Z_{\mathrm{n}}=\Delta Z_{\mathrm{i}}
$$

thus making $\Delta Z_{\mathrm{n}} / \Delta H_{\mathrm{n}}\left(=R_{\mathrm{n}}\right)$, a measure of induction effect in the vicinity of the recording site. The statistically averaged values of $R_{\mathrm{n}}$ and $R_{\mathrm{d}}$ (the daytime $\Delta Z / \Delta H$ ratio, i.e., $R_{\mathrm{d}}=\Delta Z_{\mathrm{d}} / \Delta H_{\mathrm{d}}$ ) were derived for all recording sites by establishing a linear relationship between $\Delta Z$ and $\Delta H$ of both night and daytimes respectively. The scatter plot in Fig. 4 illustrates this relationship for four representative sites characterized by high, medium and low values of $R_{\mathrm{n}}$ and $R_{\mathrm{d}}$ designated by the slopes of the best fit line. The spatial characteristics of both $R_{\mathrm{n}}$ 's and $R_{\mathrm{d}}$ 's are shown in terms of contour plots in Figs. 5(a) and 5(b) respectively. The nature of the fit for daytime events still validates the linear relationship between $\Delta Z$ and $\Delta H$, but it should be noted that during daytime, in the presence of non-uniform source, $\Delta Z_{\mathrm{d}}$ can be considered to be as

$$
\Delta Z_{\mathrm{d}}=\Delta Z_{\mathrm{e}}+\Delta Z_{\mathrm{i}}+\Delta Z^{*}
$$

where $\Delta Z_{\mathrm{e}}$ denotes the external field component arising due to non-uniformity of the source field. $\Delta Z_{\mathrm{i}}$ is the internal part of $\Delta Z$, which would result in association with the uniform source field of intensity proportional to the strength of daytime $\Delta H$ (i.e. $\Delta H_{\mathrm{d}}$ ) recorded at respective sites. This can be approximated as

$$
\Delta Z_{\mathrm{i}}=R_{\mathrm{n}} \cdot \Delta H_{\mathrm{d}}
$$

The last term, $\Delta Z^{*}$ can be regarded as a component attributable to the change in the intensity of the internal induced currents due to non-uniformity of the source field.

It follows from (1) and (2a) that

$$
\Delta Z_{\mathrm{r}}=\Delta Z_{\mathrm{e}}+\Delta Z^{*}
$$

where $\Delta Z_{\mathrm{r}}$, defined as the residual vertical field is given by

$$
\Delta Z_{\mathrm{r}}=\Delta Z_{\mathrm{d}}-R_{\mathrm{n}} \cdot \Delta H_{\mathrm{d}}
$$

It is clear that in order to find quantitatively the modulation of induction effects due to non-uniformity 

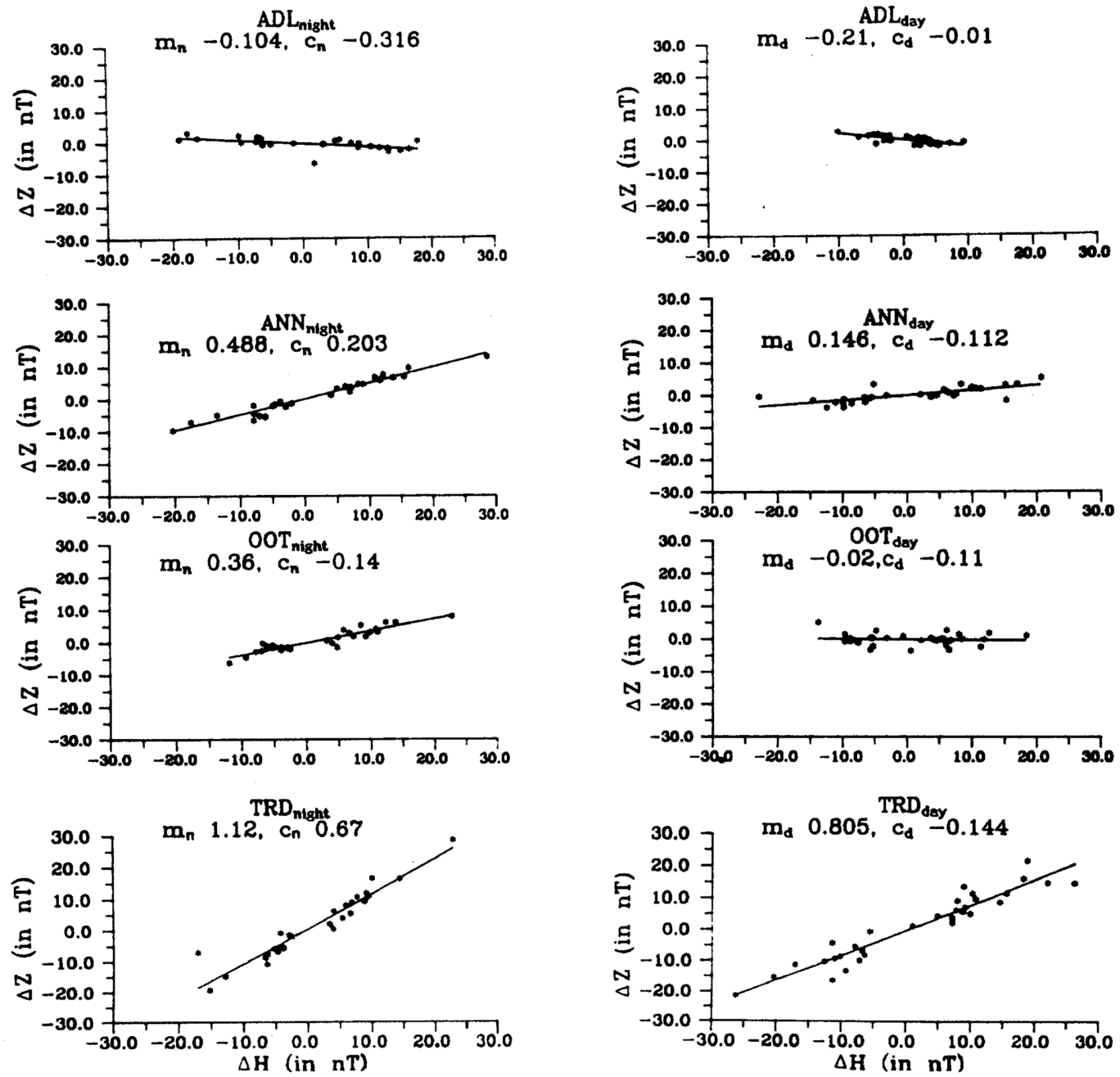

Fig. 4. Scatter plot showing relationship between vertical $(\Delta Z)$ and horizontal $(\Delta H)$ short period variations for four sites representative of equatorial (TRD), periphery of equatorial electrojet (OOT, ANN) and off the limits of equatorial electrojet (ADL) regions.

of the source (i.e. $\Delta Z^{*}$ ), it becomes necessary first to estimate and remove the external part, resulting again from the non-uniform character of the source in $\Delta Z_{\mathbf{r}}$. The applicability of the commonly employed 2-D Kertz operator to separate the electrojet field into external and internal parts (Onwumechilli, 1967; Mayaud, 1974) along the southern region of the Indian sector was questioned by Singh (1977). He argued that due to the complex internal conductivity distribution in the southern region of peninsular India, the strong anomalies exist also in $Y$-component and thus invalidate the 2-D approximation. Largely because of the only partial success of numerical schemes to decompose the observed variations into its constituents either due to the forced simplicity of models or due to complexity of the region of observations (Mareschal, 1986), much of the attempts to quantify the source effect observationally have been restricted to assess qualitatively the nature of source field bias with a view to define the treatment of data that will minimize these source effects (Beamish, 1979, 1980; Gough and de Beer, 1980; Jones, 1981). The theoretical consideration is, that $\Delta Z_{\mathrm{e}}$ and $\Delta Z^{*}$ respond differently to varying orders of spatial derivatives of the source 

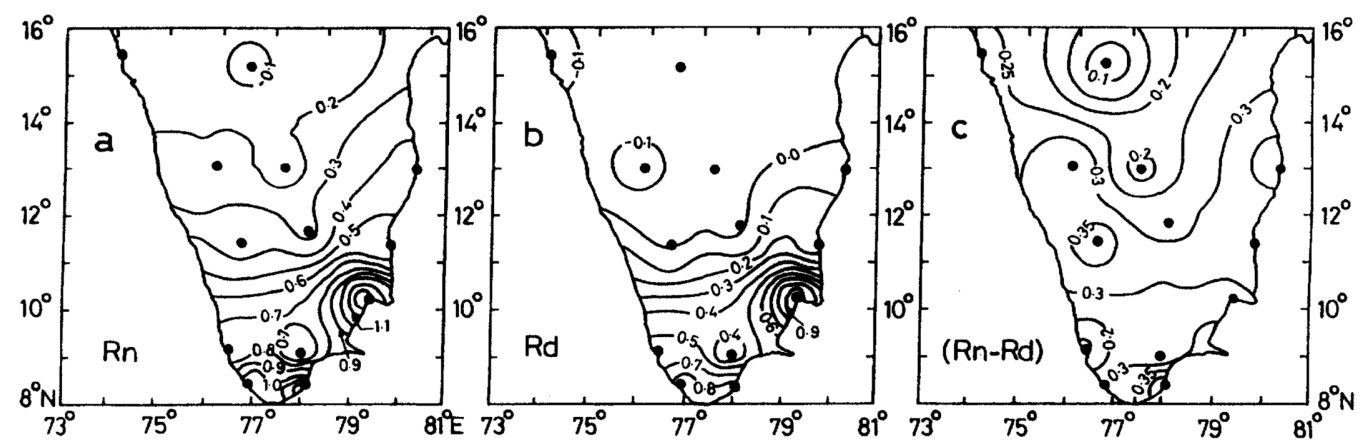

Fig. 5. Contour plots depicting the characteristics of (a) $R_{\mathrm{n}}$, (b) $R_{\mathrm{d}}$ and (c) their difference (i.e. $R_{\mathrm{n}}-R_{\mathrm{d}}$ ).

Dip Lat.

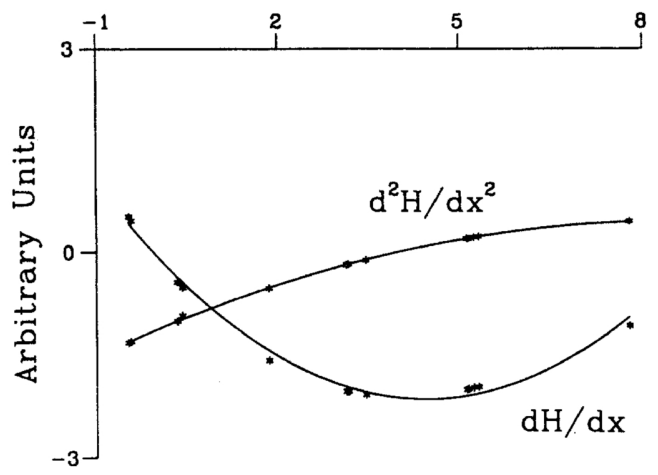

Fig. 6. Spatial characteristics of the first and second order derivatives as a function of dip latitude, for the daytime event shown in Fig. 3(a).

field and hence the dependence of $\Delta Z_{\mathrm{r}}$ on the spatial derivatives of source field was examined at all the sites by least-squares sense by obtaining a fit to the following equations

$$
\begin{aligned}
& \Delta Z_{\mathrm{r}}=b_{0}+b_{1}\left(\mathrm{~d} \Delta H_{\mathrm{d}} / \mathrm{d} x\right), \\
& \Delta Z_{\mathrm{r}}=c_{0}+c_{1}\left(\mathrm{~d} \Delta H_{\mathrm{d}} / \mathrm{d} x\right)+c_{2}\left(\mathrm{~d}^{2} \Delta H_{\mathrm{d}} / \mathrm{d} x^{2}\right)
\end{aligned}
$$

and

$$
\Delta Z_{\mathrm{r}}=d_{0}+d_{1}\left(\mathrm{~d} \Delta H_{\mathrm{d}} / \mathrm{d} x\right)+d_{2}\left(\mathrm{~d}^{2} \Delta H_{\mathrm{d}} / \mathrm{d} x^{2}\right)+d_{3}\left(\mathrm{~d}^{3} \Delta H_{\mathrm{d}} / \mathrm{d} x^{3}\right)
$$

where $\Delta H_{\mathrm{d}}$ is reckoned as a measure of intensity of the source field, whose latitudinal $(x)$ dependence for each daytime event was expressed by a polynomial of 4th degree as

$$
\Delta H_{\mathrm{d}}=a_{0}+a_{1} x+a_{2} x^{2}+a_{3} x^{3}+a_{4} x^{4}
$$


Table 1. Percentage variances of the residual vertical field $\Delta Z_{\mathrm{r}}$ accounted for by the spatial derivatives of increasing order.

\begin{tabular}{|c|c|c|c|c|c|}
\hline \multicolumn{2}{|r|}{ Station } & \multirow[t]{2}{*}{ Dip lat. } & \multicolumn{3}{|c|}{$\begin{array}{l}\text { Percentage variance of } \Delta Z_{\mathrm{r}} \text { by the } \\
\text { polynomial of }\end{array}$} \\
\hline Code & Name & & I order & II order & III order \\
\hline TRD & TRIVANDRUM & -0.42 & 8 & 63 & 69 \\
\hline TIR & TIRUCHENDUR & -0.46 & 2 & 35 & 36 \\
\hline KAY & KAYAMKULAM & 0.44 & 20 & 49 & 50 \\
\hline ETT & ETTIYAPURAM & 0.36 & 36 & 49 & 51 \\
\hline ADR & ADIRAMPATTINAM & 1.86 & 32 & 34 & 39 \\
\hline OOT & OOTACAMUND & 3.17 & 83 & 83 & 85 \\
\hline SAL & SALEM & 3.46 & 75 & 75 & 81 \\
\hline ANN & ANNAMALAINAGAR & 3.14 & 83 & 83 & 88 \\
\hline HAS & HASSAN & 5.15 & 71 & 74 & 82 \\
\hline BAN & BANGALORE & 5.12 & 54 & 56 & 60 \\
\hline MAD & MADRAS & 5.24 & 74 & 75 & 82 \\
\hline MAR & MARGAO & 5.32 & 36 & 44 & 51 \\
\hline BEL & BELLARY & 7.79 & 10 & 28 & 33 \\
\hline
\end{tabular}

It may be emphasized here, that while estimating $\Delta Z_{\mathrm{r}}$ (Eq. (2c)), the internal part compatible with uniform source field (Eq. (2a)) has already been subtracted and therefore, the least squares fitting in Eqs. (3a)-(3c) would highlight the dependence of constituents of $\Delta Z_{\mathrm{r}}$ (Eq. (2b)) which results only due to the non-uniformity of the source field. Further, it may be pertinent to use normal horizontal field $\left(H_{\text {nor }}\right)$ than the total horizontal field $\Delta H_{\mathrm{d}}$, to define the source characteristics in Eq. (4). But, as $H_{\mathrm{a}} \ll<H_{\text {nor }}$ and is randomly distributed with respect to the latitude, their presence is not expected to bias the estimate of source characteristics through the polynomial representation of the type of Eq. (4).

Figure 6 displays the spatial characteristics of the first and second derivatives as a function of diplatitude, obtained by differentiating Eq. (4) with respect to $x$, for the daytime event shown in Fig. 3(a). This decomposition indicates that the source field is characterized by the large linear gradients near the periphery of the electrojet $\left(\approx 4^{\circ}\right.$ dip latitude). In contrast to this, near the peak of the source current, i.e., near the dip equator, the second order spatial derivative $\left(\mathrm{d}^{2} \Delta H_{\mathrm{d}} / \mathrm{d} x^{2}\right)$ is dominant though its maximum value is small compared to the largest value of linear gradient near the periphery of the electrojet belt.

The percentage variances of $\Delta Z_{\mathrm{r}}\left(\Delta Z_{\mathrm{r}}{ }^{\text {obs }}\right)$ accounted for by the polynomial of a given order was computed using the formula

$$
\left(1-\left(\sum_{i}^{n}\left(\Delta Z_{\mathrm{r}}^{\mathrm{obs}}-\Delta Z_{\mathrm{r}}^{\mathrm{cal}}\right)^{2} / \sum_{i}^{n}\left(\Delta Z_{\mathrm{r}}^{\mathrm{obs}}\right)^{2}\right)\right) \times 100 .
$$

Where $\Delta Z_{\mathrm{r}}$ cal is the calculated value of $\Delta Z_{\mathrm{r}}$ obtained by substituting the derived coefficients in respective equations (3a), (3b) or (3c). $n$ is the total number of events used. The so computed percentage variances accounted for by the polynomial of increasing order (Eqs. (3a) through (3c)) are given in Table 1 for all the stations.

\section{Discussion}

\subsection{Spatial behavior of $R_{n}$ : Induction effect}

During nighttime, since $\Delta Z$ variations are primarily of internal origin, the spatial variations of $R_{\mathrm{n}}$ (Fig. $5(\mathrm{a}))$ exhibit a character of internal conductivity distribution. The values of $R_{\mathrm{n}}$ show quite a good agree- 
ment with the magnitude of statistically derived induction arrows (Thakur et al., 1986). Discussing the conductivity anomalies of south India, several authors (Papamastorakis and Haerendel, 1983; Singh and Agarwal, 1983; Ramaswamy et al., 1985; Thakur et al., 1986; Mareschal et al., 1987; Agarwal and Weaver, 1989) have deduced the qualitative and quantitative estimates of conductivity distribution compatible with the observed induction pattern. The $R_{\mathrm{n}}$ 's at all stations located along the coast line are marginally higher compared to the inland stations. This can be attributed to the conventional coast effect. The relatively large values of $R_{\mathrm{n}}$ along the east coast compared to the west coast were shown to be largely determined by the regionally induced internal currents channeled through the deep seated conductor probably located in the upper mantle or lower crust between India and Sri Lanka (Rajaram et al., 1979; Singh and Agarwal, 1983; Thakur et al., 1986). The dominance of current channeling was first indicated by Nityananda et al. (1977) from the direction of induction arrows at ANN, which they showed to be pointing southward, rather than at right angles to the continental shelf, clearly depicting the changes in the direction of flow path of induced currents in the sea as they approached the coast. The abnormally large values of $R_{\mathrm{n}}$ all along the southern tip of peninsular India is a manifestation of the effect of current concentration in multiple conductors. These conductors have direct relevance to the triple junction formed between the Indo-Ceylon Graben (aligned with Palk-Strait), the Comorin ridge and the west coast rift (Ramaswamy et al., 1985; Thakur et al., 1986; Mareschal et al., 1987; Agarwal and Weaver, 1989). The $R_{\mathrm{n}}$ 's in excess of 1.0 at TIR and ADR are attributed to the favorable location of these stations with respect to the channeled currents through Palk-Strait conductor.

\subsection{Spatial behavior of $R_{d}$ : Source field effect}

From the point of view to isolate the influence of source field effect on induction process, the feature of interest is the relative changes in $R_{\mathrm{n}}$ and $R_{\mathrm{d}}$. Examination of Figs. 5(a) and 5(b) clearly reveals that $R_{\mathrm{d}}$ at each station is relatively small compared to their corresponding nighttime counterpart. The spatial characteristics of the relative changes between $R_{\mathrm{n}}$ and $R_{\mathrm{d}}$ have become more apparent when their differences were contoured separately as shown in Fig. 5(c). Superimposed on the relatively large differences all along the coast line, two zones of pronounced differences are seen in Fig. 5(c). One is centered around the periphery of the equatorial electrojet (Zone I) and the other around the central axis of the electrojet (Zone II). The extent to which the observed reduction of $R_{\mathrm{d}}$ 's compared to $R_{\mathrm{n}}$ 's in these two zones manifests the influence of external contribution to $Z$ or the modulation of flow of internal induced currents, both resulting from the non-uniformity of the source, is evaluated taking into consideration the flow pattern of internal currents as well as spatial behavior of source-current as characterized by the first three spatial derivatives.

\subsubsection{The quenching effect (Zone I)}

The latitudinal behavior of $\Delta H$ during daytime (Fig. 3(a)) is similar to those seen in association with the augmentation of quiet-day solar daily $(S q)$, variations in the electrojet belt. The latitudinal behavior of magnetic field variations associated with electrojet like band of current has been examined by several workers (e.g. see Richmond, 1973). It is simple to visualize that the negative latitudinal gradients associated with such concentrated eastward directed current would yield upward (negative) $\Delta Z$ at all northern low-latitude stations with maximum magnitude at sites corresponding to largest gradient (i.e. near the periphery of the electrojet). As against this, the positive values of $R_{\mathrm{n}}$ 's at all stations suggest that the internal currents associated with the eastward directed inducing currents produce downward (positive) $\Delta Z$ at all stations. Thus, the partial reduction of $R_{\mathrm{d}}$ 's may be a manifestation of the mutual cancellation of opposing external and internal parts in $\Delta Z$ component. Such a mechanism was earlier invoked by Papamastorakis and Haerendel (1983) and Singh and Agarwal (1983) to explain the disappearance of $\Delta Z$ variations accompanying strong $\Delta H$ perturbations at Annamalainagar (ANN) during daytime. Our results clearly show that this quenching effect appears to be complete all along OOT, SAL and a little north of ANN where the $R_{\mathrm{d}}$ 's reduce to zero (Fig. 5(b)). This inference is corroborated by the relationship between $\Delta Z$ and the spatial derivatives. As seen in Table 1 , as much as 90 percent of variance of residual vertical field, $\Delta Z_{\mathrm{r}}$, at OOT, SAL and ANN is accounted for by the linear gradients in the source field. As the second 
or higher spatial derivative, which controls the source field effect on the induction process, contribute negligibly to $\Delta Z_{\mathrm{r}}$, that large and significant differences in $R_{\mathrm{n}}$ and $R_{\mathrm{d}}$ ratios at stations close to the periphery of the electrojet can be attributed to the quenching of internal field $\left(\Delta Z_{\mathrm{i}}\right)$ by external part $\left(\Delta Z_{\mathrm{e}}\right)$, the later resulting from the linear gradient in non-uniform source field.

4.2.2. Modulation of induced currents intensity (Zone II)

The second zone, where $R_{\mathrm{n}}$ 's and $R_{\mathrm{d}}$ 's differ significantly is located around the central axis of the electrojet. For the group of stations, which fall under this zone viz., TRD, TIR and KAY the total variance of $\Delta Z_{\mathrm{r}}$ accounted for by the polynomial including spatial derivatives of third order range between 35-70 percent (Table 1) against the 90 percent variance accounted for by polynomial of same order near the periphery of the electrojet. At the site ADR, located in between the two zones of pronounced differences in $R_{\mathrm{n}}$ and $R_{\mathrm{d}}$, the overall variance largely controlled by the first derivative is also small, $(\approx 40)$ recall that major induction effects at ADR, TIR and TRD are controlled by the channeling of currents through a conductor beneath the Palk-Strait. In so far as channeling is concerned its contribution, being determined by the induction process operating on regional scale, would be less controlled by the spatial derivatives of localized electrojet source current. The fluctuating strengths of these channeled currents may account for the low variance of $\Delta Z_{\mathrm{r}}$ accounted for by the spatial derivative.

Examination of latitudinal profile of $\Delta H_{\mathrm{d}}$ (Fig. 3(a)) and its decomposed spatial derivative (Fig. 6) shows that the latitudinal gradients of the source field near the electrojet axis are much smaller than the gradients seen near the periphery of the electrojet. In view of these observational features, the net reduction of $R_{\mathrm{d}}$ 's near the electrojet axis, which in magnitude are comparable to those seen near the periphery of the electrojet, can not be attributed to the sole quenching effect between internal and external fields, but some other mechanism may also concurrently be operative. It is noteworthy that in the narrow latitude band centered at dip equator, it is the second derivative which is dominant accounting for the largest part of the variance (Table 1). The contribution of the third derivative to the total variance is negligible. Consistent with the theory, it provides a strong observational evidence that at least a fractional reduction of $R_{\mathrm{d}}$ near the center of localized electrojet currents arise from the weakening of the induced currents related to the presence of second order spatial derivatives in the electrojet source current. The relatively important weakening of $R_{\mathrm{d}}$ 's at coastal stations compared to distant land stations is in agreement with the results of Hermance (1984) who showed that the field induced by the localized source polarized parallel to the strike are perturbed only in the close vicinity of the lateral inhomogeneity.

\section{Conclusion}

With the help of contour plots of $\Delta Z / \Delta H$ ratios for day and nighttimes, the effect of source field nonuniformity on geomagnetic induction is highlighted. Two zones of significant differences between day and nighttime ratios were found to lie near the central axis and periphery of equatorial electrojet. In the later zone, the decrease in daytime $\Delta Z / \Delta H$ ratios is due to the mutual balance of external and internal parts of vertical component. In the former, the reduction in daytime $\Delta Z / \Delta H$ ratios is found to be related largely to the second order spatial derivatives of the source field. In agreement with the theoretical models, this indicates the weakening of intensity of induced currents near the axis of the non-uniform source where the second derivatives dominate the spatial character of the inducing source. Results also indicate that the source field effect tends to be dominant near the lateral conductivity contrast.

We are very much grateful to Prof. B. P. Singh, our director, for many useful discussions. We would like to express our sincere thanks to Dr. M. Mareschal and an anonymous referee for their valuable comments which have improved the quality of the paper. Mr. Shelatkar and Mr. Waknis have drafted Figs. 1 and 5.

\section{REFERENCES}

Agarwal, A. K. and J. T. Weaver, Regional electromagnetic induction around the Indian peninsula and Sri Lanka: A three 
dimensional numerical study using thin sheet approximation, Phys. Earth Planet. Inter., 54, 320-331, 1989.

Agarwal, A. K. and J. T. Weaver, A three-dimensional numerical study of regional electromagnetic induction in Southern India by an electrojet source, Phys. Earth Planet. Inter., 60, 1-17, 1990.

Beamish, D., Source field effects on transfer functions at mid-latitudes, Geophys. J. Roy. Astr. Soc., 58, 117-134, 1979.

Beamish, D., Diurnal characteristics of transfer functions at pulsation periods, Geophys. J. Roy. Astr. Soc., 61, 623-643, 1980.

Berdichevsky, M. N., V. I. Dimitriev, and U. Schmucker, Generalized magnetotelluric and magnetic gradient relations for a layered half-space, Presented at IAGA General Assembly, Edinburgh, August, 3-15, 1981.

Dimitriev, V. I. and M. N. Berdichevsky, The fundamental model of magnetotelluric sounding, Proc. IEEY, 67, $1034-1043,1979$.

Gough, D. I. and J. D. de Beer, Source field bias in geomagnetic transfer functions, J. Geomag. Geoelectr., 32, 1471-1482, 1980.

Hermance, J. F., Electromagnetic induction by finite wavenumber source fields in 2-D lateral heterogeneities: The transverse electric mode, Geophys. J. Roy. Astr. Soc., 78, 159-179, 1984.

Jones, A. G., Geomagnetic induction studies in Scandinavia. II-Geomagnetic depth sounding, induction vectors and coast effect, J. Geophys., 50, 23-26, 1981.

Mareschal, M., Source field effects and the interpretation of geomagnetic depth sounding data at sub-auroral latitudes, Geophys. J. Roy. Astr. Soc., 67, 125-136, 1981.

Mareschal, M., Modelling of natural sources of magnetospheric origin in the interpretation of regional induction studies: A review, Surv. Geophys., 8, 261-300, 1986.

Mareschal, M., G. Vasseur, B. J. Srivastava, and R. N. Singh, Induction models of southern India and the effect of off-shore geology, Phys. Earth Planet. Inter., 45, 137-148, 1987.

Mayaud, P. N., About the effects induced by the daily variation due to the equatorial electrojet, J. Atmos. Terr. Phys., 36, 1367$1376,1974$.

Nityananda, N., A. K. Agarwal, and B. P. Singh, Induction at short periods in the horizontal variations in the Indian peninsula, Phys. Earth Planet. Inter., 15, 5-9, 1977.

Onwumechilli, A., Geomagnetic variations in the equatorial zones, in Physics of Geomagnetic Phenomena, Vol. 1, edited by S. Matsushita and W. H. Campbell, pp. 425-507, Acad. Press, New York, 1967.

Papamastorakis, I. and G. Haerendel, A possibility to distinguish between ionospheric and magnetospheric origin of low latitude magnetic perturbations, J. Geophys., 52, 53-60, 1983.

Rajaram, M., B. P. Singh, N. Nityananda, and A. K. Agarwal, Effect of the presence of a conducting channel between India and Sri Lanka Island on the features of equatorial electrojet, Geophys. J. Roy. Astr. Soc., 56, 127-138, 1979.

Ramaswamy, V., A. K. Agarwal, and B. P. Singh, A three dimensional numerical model study of electromagnetic induction around the Indian peninsula and Sri Lanka island, Phys. Earth Planet. Inter., 39, 52-61, 1985.

Rastogi, S., B. R. Arora, and B. P. Singh, Source field geometry and electromagnetic induction in the Earth: Some observational results from India, Ann. Geophys., 4, B-5, 529-536, 1986.

Reddy, C. D., Qualitative appraisal of the modified Tikhonov-Cagniard equation in electromagnetic induction, Ind. J. Rad. Space Phys., 21, 366-369, 1992.

Richmond, A. D., Equatorial electrojet-II-Use of model to study the equatorial ionospheric, J. Atmos. Terr. Phys., 38, 1105$1118,1973$.

Schmucker, U., Anomalies of geomagnetic variations in south western United States, Bull. Scripps Instt. Oceangr. Tech. Ser., $13,1970$.

Singh, B. P., Subsurface structure and the equatorial electrojet, Proc. Workshop on Equatorial Electrojet and Associated Phenomena, pp. 39-50, Physical Research Laboratory, Ahmedabad, India (October 25-29), 1977.

Singh, B. P. and A. K. Agarwal, On the spatial and temporal separation of geomagnetic anomalies at the southern tip of Indian peninsula, Phys. Earth Planet. Inter., 31, 59-64, 1983.

Singh, B. P., A. K. Agarwal, and L. Carlo, Anomalies in $H$ at Indian equatorial stations and their effect on equatorial enhancements, J. Atmos. Terr. Phys., 44, 241-244, 1982.

Thakur, N. K., M. V. Mahashabde, B. R. Arora, B. P. Singh, B. J. Srivastava, and S. N. Prasad, Anomalies in geomagnetic variations on peninsular India near Palk Strait, Geophys. Res. Lett., 8, 947-950, 1981.

Thakur, N. K., M. V. Mahashabde, B. R. Arora, B. P. Singh, B. J. Srivastava, and S. N. Prasad, Geomagnetic variation analysis in peninsular India. Geophys. J. Roy. Astr. Soc., 86, 839-854, 1986.

Wait, J. R., On the relation between telluric currents and the earth's magnetic field, Geophysics, 19, 281-289, 1954. 